\title{
Quantum Ergodicity on the Sphere
}

\author{
Steven Zelditch \\ Department of Mathematics, The Johns Hopkins University, Baltimore, MD 21218, USA
}

Received March 23, 1990; in revised form October 10, 1991

Abstract. We prove that a random orthonormal basis of eigenfunctions on the standard sphere has quantum ergodic behavior.

As the title portends, this paper is about quantum ergodicity in the most completely integrable of examples: the Laplacian on $S^{2}$. The notion of quantum ergodicity we pursue here is the one which characterizes ergodicity of a Schrödinger (or Laplace) operator $H$ in terms of the semi-classical behaviour of its eigenfunctions ([Sn, V, $\mathrm{Be}, \mathrm{Z} .1-2$, CdeV.2, HMR, ST]). Roughly, $H$ is quantum ergodic if its orthonormal bases $\left\{\varphi_{j}\right\}$ of eigenfunctions have the following property: $\left(A \varphi_{j}, \varphi_{j}\right) \rightarrow \int_{S^{*} M} \sigma_{A} d \mu$ $(j \rightarrow \infty)$ for any $0^{\text {th }}$ order $\Psi D O A\left(d \mu=\right.$ Liouville measure, $\sigma_{A}=$ principal symbol). This limit formula is a kind of quantum analogue of the Birkhoff ergodic theorem and is known to hold whenever the classical (e.g. geodesic) flow is ergodic. Otherwise it does not seem well understood: for example, it might (for all that is proved to date) even hold for a generic Laplacian. Our purpose here is, perversely, to investigate it on the sphere. Of course, the usual basis $\left\{Y_{m}^{l}\right\}$ of spherical harmonics does not have the ergodic property. But, due to the high degeneracy of eigenvalues, there is an infinite dimensional manifold of orthonormal bases of eigenfunctions. This manifold is actually a group and carries a unit mass Haar measure. Our main result is that, relative to this measure, almost all bases have the ergodic property.

To state the result more precisely we will need to introduce some terminology and background.

Throughout this paper we will be considering only the standard 2-sphere $S^{2}$, although our methods would work on many other spaces. We will usually omit explicit reference to the metric on $S^{2}$; all notation such as $L^{2}\left(S^{2}\right), \Delta$, etc., will refer to the standard metric.

We first recall that

$$
L^{2}\left(S^{2}\right)=\bigoplus_{l} E_{l} \quad\left(\operatorname{dim} E_{l}=2 l+1\right)
$$

where $E_{l}$ is the complex eigenspace of spherical harmonics of degree $l$. Equivalently, $E_{l}$ is the eigenspace of the laplacian $\Delta$ of eigenvalue $l(l+1)$. We will let $\pi_{l}$ denote 
the orthogonal projection onto $E_{l}$ :

$$
\pi_{l}: L^{2}\left(S^{2}\right) \rightarrow E_{l}
$$

Next, recall that a (hermitian) orthonormal basis for $E_{l}$ is provided by the vectors $\left\{Y_{m}^{l}, m=-l, \ldots, l\right\}$, determined (up to constants) by the conditions;

$$
\left\{\begin{array}{l}
\Delta Y_{m}^{l}=l(l+1) Y_{m}^{l} \\
(1 / i) \partial / \partial \theta Y_{m}^{l}=m Y_{m}^{l},
\end{array}\right.
$$

where $\partial / \partial \theta$ generates rotation around the $Z$-axis. See, for example, [T, 2.1] for the specific constants.

The basis $\left\{Y_{m}^{l}, l=0,1,2, \ldots ; m=-l, \ldots, l\right\}$ has some very special asymptotic properties as $l \rightarrow \infty$, reflecting the complete integrability of the geodesic flow on $S^{2}$. Indeed, for any fixed rational $e$, let $\mathscr{H}_{e}$ be the subspace of $L^{2}\left(S^{2}\right)$, spanned by $\left\{Y_{m}^{l}: m / l=e\right\} . \mathscr{H}_{e}$ is the quantum analogue of the invariant torus $T_{e} \subset S^{*}\left(S^{2}\right)$ for the geodesic flow, consisting of all great circles whose angle $\phi$ with the $Z$-axis satisfies: $\cos \phi=e$. The precise meaning of "analogue" may be stated in several ways:

(i) $T_{e}$ is the microsupport of the quasi-mode $\mathscr{H}_{e}$ (more precisely, the cone through $T_{e} ;$ see $[\mathrm{C}-\mathrm{de} \mathrm{V}]$ );

$$
\lim _{\substack{l \rightarrow \infty \\ m / l=e}}\left(A Y_{m}^{l}, Y_{m}^{l}\right)=\int_{T_{e}} \sigma_{A} d \mu_{e}
$$

where $A$ is a $0^{\text {th }}$ order pseudo-differential operator with symbol $\sigma_{A}$, and where $d \mu_{e}$ is the invariant probability measure on $T_{e}$ for the torus action determined by the geodesic flow and by rotation about the $z$-axis (see [Z.1]);

(iii) $\mathscr{H}_{e}$ is the ladder subspace corresponding to the co-isotropic cone $\Phi^{-1}(\{r(m, l)$ : $\left.m / l=e, r \in \mathbb{R}^{+}\right\}$), where $\Phi$ is the moment map for the above torus action ( $\left.[\mathrm{G} \cdot \mathrm{S}]\right)$.

Thus, the foliation of $S^{*}\left(S^{2}\right)$ by invariant torii $T_{e}$ for the geodesic flow has for quantum analogue the decomposition of $L^{2}$ into ladders $\mathscr{H}_{e}$, invariant under $\Delta$; sequences of eigen-functions in $\mathscr{H}_{e}$ concentrate, in the limit of high eigenvalues, on $T_{e}$; or, equivalently, functions in $\mathscr{H}_{e}$ can only have their wave front sets in the cone thru $T_{e}$.

The main object of the present paper is to contrast these asymptotic properties of the "completely integrable orthonormal basis" $\left\{Y_{m}^{l}\right\}$ with the properties of a "random" orthonormal basis of Laplace eigenfunctions. It turns out that the random basis behaves like an orthonormal basis of eigenfunctions on a Riemannian manifold with ergodic geodesic flow.

Let us recall this ergodicity of eigenfunctions ( $[\mathrm{Sn}, \mathrm{Z} .2,(\mathrm{CdV} .2)])$. To begin with, let $(M, g)$ be any compact, Riemannian manifold, let $G^{t}$ be the geodesic flow on $S^{*} M$, let $\Delta$ be the Laplacian and let $\left\{\varphi_{j}\right\}$ be an orthonormal basis of Laplace eigenfunctions: $\Delta \varphi_{j}=\lambda_{j} \varphi_{j}$. Let us say:

(1.5) Definition. $\left\{\varphi_{j}\right\}$ is a Liouville-distributed basis if, for any YDO (pseudodifferential operator) $A$ of order 0 ,

$$
\lim _{\lambda \rightarrow \infty} \frac{1}{N(\lambda)} \sum_{\sqrt{\lambda_{J}} \leqq \lambda}\left|\left(A \varphi_{j}, \varphi_{j}\right)-\bar{\sigma}_{A}\right|^{2}=0 .
$$


Here, $N(\lambda)=\#\left\{j: \sqrt{\lambda_{j}} \leqq \lambda\right\},\left(A \varphi_{j}, \varphi_{j}\right)$ is the matrix-coefficient of the $\Psi D O A$, and $\bar{\sigma}_{A}=\frac{1}{\operatorname{vol}\left(S^{*} M\right)} \int_{S^{*} M} \sigma_{A} d \mu$, where $d \mu$ is the Liouville measure on $S^{*} M$.

To understand (1.6), we recall a standard lemma on bounded sequences $\left\{a_{j}\right\}$ in $\mathbb{C}([\mathrm{Wa}])$.

This says:

iff

$$
\lim _{N}(1 / N) \sum_{j \leqq N}\left|a_{j}\right|^{p}=0 \quad(\text { any } p>0)
$$

$$
\begin{aligned}
& \lim _{k \rightarrow \infty} a_{j_{k}}=0, j_{k} \in \mathscr{S} \subset \mathbb{Z}^{+}, \text {where } \mathscr{S}=\left\{j_{k}\right\} \text { is a subsequence of } \mathbb{Z}^{+} \text {of } \\
& \text { density } 1: 1 / N \#\left\{j_{k} \in \mathscr{S}\right\} \cap\{1,2, \ldots, N\} \rightarrow 1 .
\end{aligned}
$$

The limit formula (1.6) is thus equivalent to the existence of a subsequence $\mathscr{S}=\left\{j_{k}\right\}$ of spectral density one (in the obvious sense) for which

$$
\left(A \varphi_{j_{k}}, \varphi_{j_{k}}\right) \rightarrow \bar{\sigma}_{A}
$$

The subsequence $\mathscr{S}$ a priori depends on $A$. However by a diagonalization argument one can show that (1.6) implies the existence of a subsequence $\mathscr{S}$ of density one for which (1.8) holds for all $A$ (see [Z.2]).

The limit formulae (1.8) and (1.6) give a quantum analogue of ergodicity. For example if $\sigma_{A}$ is the characteristic function of a nice subset $E \subset S^{*} M$, then $\left(A \varphi_{j}, \varphi_{j}\right)$ is interpreted as the probability that a free particle in state $\varphi_{j}$ has its (position, momentum) in $E$. As the energy $\lambda_{j}$ tends to infinity, this should tend to the probability that a free classical particle, i.e. a geodesic, goes through $E$. This probability, calculated according to (1.8), is just the Liouville measure of $E$.

This heuristic reasoning suggests that if the geodesic flow $G^{t}$ is ergodic, then (1.8) should hold for any orthonormal basis of eigenfunctions. Indeed, this is the case ([Sn, Z.2, CdV]). Conversely, if (1.8) holds for any orthonormal basis of eigenfunctions, then the geodesic flow should be ergodic. This converse direction seems however to be quite difficult. Our main result in this paper implies that, if we replace the "any" by "some" or even "almost any," then in fact the converse is false. Indeed, we will show that the random orthonormal basis of eigenfunctions on $S^{2}$ is Liouville distributed.

It emerges that existence of a Liouville-distributed basis of eigenfunctions for a Laplacian $\Delta$ is not a good definition of the "quantum ergodicity" of $\Delta$. It seems to us that a more reasonable criterion for quantum ergodicity is in terms of operator time-averages, where $\bar{A}=w-\lim _{T \rightarrow \infty} \frac{1}{2 T} \int_{-T}^{T} e^{-i t \sqrt{\Delta}} A e^{i t \sqrt{\Delta}} d t$ is the time average of $A$. The criterion is: $\Delta$ (or $e^{i t \sqrt{\Delta}}$ ) is ergodic if, for all $\psi$ DO's $A$ or order $0, \bar{A}=\bar{\sigma}_{A}(\mathrm{Id})+K$, where the Hilbert-Schmidt norm of $\pi_{\lambda} K \pi_{\lambda}$ is $o(N(\lambda))$ as $\lambda \rightarrow \infty\left(\pi_{\lambda}\right.$ is the spectral projection for the interval $[0, \lambda]$ and $N(\lambda)=\operatorname{tr} \pi_{\lambda}$. In $[\mathrm{Z} 1-\mathrm{Z} 2]$, we showed that $\Delta$ is ergodic in this sense if the geodesic flow is ergodic; such ergodicity also implies that all orthonormal bases are Liouville distributed. It is not yet clear to us if this ergodicity of $\Delta$ implies classical ergodicity.

Returning to our main result, we first note that the set $\mathscr{O B}$ of orthonormal basis of Laplace eigenfunctions of $S^{2}$ is a probability space. Indeed, any orthonormal 
basis $\left\{\varphi_{m}^{\ell}: m=-\ell, \ldots, \ell\right\}$ of $E_{\ell}$ corresponds to an element $\tau_{\ell} \in U(2 \ell+1)$ via:

$$
\varphi_{m}^{\ell}=\tau_{\ell} Y_{m}^{\ell}
$$

It follows that the manifold of orthonormal bases of $E_{\ell}$ can be identified via (1.9) with the unitary group $U(2 l+1)$. Hence, the infinite dimensional manifold of orthonormal bases of eigenfunctions can be identified with the product:

$$
\mathcal{O} \mathscr{B} \cong U(1) \times U(3) \times U(5) \times \cdots .
$$

Now each $U(2 \ell+1)$ carries a Haar measure $d \mu_{\ell}$, normalized to have mass one. Hence $\mathcal{O O B}$ carries the probability measure

$$
d \mu_{\infty}=d \mu_{1} \times d \mu_{3} \times \cdots .
$$

Let us denote by $\tau_{\infty}$ the sequence $\left(\tau_{1}, \tau_{2}, \tau_{3}, \cdots\right)$. Let us say, as in (1.6)-(1.8), that $\tau_{\infty}$ is Liouville distributed,

$$
\tau_{\infty} \in \mathscr{L} \mathcal{O} \mathscr{B},
$$

if, for any $\Psi D O A$ of order 0 ,

$$
\lim _{L \rightarrow \infty}(1 / L) \sum_{\ell \leqq L}\left(\frac{1}{2 \ell+1} \sum_{m=-\ell}^{\ell}\left|\left(\pi_{\ell} A \pi_{\ell} \tau_{\ell} Y_{m}^{\ell}, \tau_{\ell} Y_{m}^{\ell}\right)-\bar{\sigma}_{A}\right|^{2}\right)=0 .
$$

Our main result is, then:

$$
\mu_{\infty}(\mathscr{L} \mathcal{O} \mathscr{B})=1 .
$$

Thus, almost every orthonormal basis of Laplace eigenfunctions on $S^{2}$ behaves ergodically.

\section{2. $\mu_{\infty}(\mathscr{L} \mathscr{O} \mathscr{B})=1$}

Let $A$ be a Hermitian $\Psi D O$ of order 0 on $L^{2}\left(S^{2}\right.$, can). Associated to $A$ is the sequence $\left\{\pi_{\ell} A \pi_{\ell}: \ell=0,1,2, \ldots\right\}$ of finite rank Hermitian operators on the sequence $\left\{E_{\ell}\right\}$ of Hermitian vector spaces. Having fixed the basis $\left\{Y_{m}^{\ell}: m=-\ell, \ldots, \ell\right\}$ of $E_{\ell}$, we can identify $E_{\ell}$ with $\mathbb{C}^{2 \ell+1}$ and $\pi_{\ell} A \pi_{\ell}$ with a $(2 \ell+1) \times(2 \ell+1)$ Hermitian matrix. Thus, $i \pi_{\ell} A \pi_{\ell}$ may be considered an element of $u(2 \ell+1)$ (the Lie algebra of $U(2 \ell+1) \cong$ $U\left(E_{\ell}\right)$. As such, it is conjugate to an element $\mu^{\ell}(A)=\left(\begin{array}{ccc}\mu_{1}^{\ell} & & \\ & \ddots & \\ & & \mu_{2 \ell+1}^{\ell}\end{array}\right)$ of the Cartan subalgebra $h_{2 \ell+1}$ of diagonal matrices in $U(2 \ell+1) \cdot \mu^{\ell}=\mu^{\ell}(A)$ is uniquely determined if we require that $\mu_{1}^{\ell} \geqq \mu_{2}^{\ell} \geqq \cdots \geqq \mu_{2 \ell+1}^{\ell}$, i.e. that $\mu^{\ell}$ lies in the positive Weyl chamber $h_{2 \ell+1,+}$ of $U(2 \ell+1)$.

Corresponding in turn to $\mu^{\ell}$ are:

i) its adjoint orbit $\mathcal{O}_{\mu}$ in $u(2 \ell+1)$;

ii) the probability measure $d m_{\mu^{\prime}}=\frac{1}{2 \ell+1} \sum_{i=1}^{2 \ell+1} \delta\left(\mu-\mu_{i}^{\ell}\right)$ on $\mathbb{R}$. 
The correspondences $\mu^{\ell} \mapsto \mathcal{O}_{\mu^{\ell}} \leftrightarrow d m_{\mu^{\prime}}$ are all 1-1. On the other hand, the correspondences $A \rightarrow\left\{\pi_{\ell} A \pi_{\ell}\right\} \rightarrow\left\{\mu^{\ell}(A)\right\}$ are not. Indeed, if $A^{\text {ave }}=\frac{1}{2 \pi} \int_{0}^{2 \pi} e^{-i t P} A e^{i t P} d t$, where $\left.P\right|_{E_{\ell}}=\ell$, then $A^{\text {ave }}=\bigoplus_{\ell} \pi_{\ell} A \pi_{\ell}$; hence $A$ and $A^{\text {ave }}$ correspond to the same sequence $\left\{\pi_{t} A \pi_{t}\right\} . A^{\text {ave }}$ is of course just the diagonal part of $A$, studied at some length in ([Wei, Wi, Gu, U]). Since the definition of $\mathscr{L} \mathscr{O} \mathscr{B}$ depends only on consideration of diagonal parts of $\Psi D O$ 's, we will henceforth assume $A=A^{\text {ave }}$. In other words, we will always assume $[A, \Delta]=0$. The bounded $\Psi$ DO's $A$ commuting with $\Delta$ form a ring, denoted $\mathscr{C}$ in [U]. With no loss of generality, we assume henceforth that $A \in \mathscr{C}$.

Even with this assumption, the correspondence $\left\{\pi_{\ell} A \pi_{\ell}\right\} \rightarrow\left\{\mu^{\ell}(A)\right\}$ is not 1-1. For example, if $A^{g}=T_{g} A T_{g}^{*}$, where $g \in S O(3)$ and $T_{g}$ is the corresponding unitary translation on $L^{2}\left(S^{2}\right)$, then obviously $\mu^{\ell}\left(A^{g}\right)=\mu^{\ell}(A)$. (It is possible however that, conversely, $\mu^{\ell}\left(A_{1}\right)=\mu^{\ell}(A)(\forall \ell)$ implies $A_{1}=A^{g}$ for some $g$.) Moreover, the correspondence is far from surjective. Indeed, the Szegö limit Theorems of WeinsteinWidom (loc. cit.) show that the sequences $\left\{\mu_{\ell}(A)\right\}$ have quite special asymptotic properties. The most important for this paper is that the measures $d m_{\mu^{\rho}}(A)$ have a weak limit $d m_{\mu_{\infty}}$ as $\ell \rightarrow \infty$ :

$$
d m_{\infty}=\sigma_{A}^{\text {ave }} * d \mu,
$$

where $d \mu$ is Liouville (i.e. Haar) measure on $S^{*}\left(S^{2}\right)$ (i.e. $S O(3)$ ), and where $\sigma_{A}^{\text {ave }}$ is the averaged principal symbol of $A$ :

$$
\sigma_{A}^{\mathrm{ave}}(x, \xi)=\frac{1}{2 \pi} \int_{0}^{2 \pi} \sigma_{A}\left(\left(G^{t}(x, \xi)\right) d t \quad\left(G^{t}=\text { geodesic flow }\right)\right.
$$

Actually (2.2) is only the first of a sequence of distributions associated to $\left\{\mu^{\ell}(A)\right\}$. Indeed, it is prove in [Wei] (see also $[\mathrm{Gu}, \mathrm{U}]$ ) that

$$
\int_{\mathbb{R}} f d m_{\mu^{\ell}(A)} \sim \sum_{j=0}^{\infty} \beta_{j}(f) \ell^{-j}
$$

for certain $\beta_{j} \in \mathscr{D}^{\prime}(\mathbb{R})$ (depending on $A \in \mathscr{S}$ ), and with $\beta_{0}=d m_{\infty}$. We will not use the higher $\beta_{j}$ 's in this paper. However, their existence constrains the sequence of orbits $\left\{\mathcal{O}_{\mu^{\ell}}\right\}$ associated to $A \in \mathscr{C}$.

We now reformulate our theorem in terms of the orbits $\left\{\mathcal{O}_{\mu^{\prime}}\right\}$.

First, we fix an Ad-invariant inner product $\langle\cdot\rangle$ on $u(2 \ell+1)$ : for example, $B_{0}(X, Y)=\operatorname{Tr} X Y$. Then let $J_{\ell}$ be the corresponding orthogonal projection: $u(2 \ell+1) \rightarrow h_{\ell}$. Thus,

$$
J_{\lambda}\left(a_{i j}\right)=\left(\begin{array}{ccc}
a_{11} & & 0 \\
& \ddots & \\
0 & & a_{2 \ell+1,2 \ell+1}
\end{array}\right) .
$$

Under the identifications $u \cong u^{*}, h \cong h^{*}$ induced by $B_{0}$, the orbits $\mathcal{O}_{\mu}$ go over to co-adjoint orbits $\mathcal{O}_{\mu}^{*}$. These are symplectic manifolds, and the maximal torus $H_{\ell}$ corresponding to $h_{\ell}$ acts on them in a Hamiltonian fashion (by conjugation). The moment map for this action can then be identified, as above, with $J_{\ell}$. 
Further, let $u(2 \ell+1) \cong \operatorname{su}(2 \ell+1) \oplus \mathbb{R}$ be the decomposition in terms of trace-less and scalar parts: $a=a^{0}+\bar{a}$, where $\bar{a}=\left(\frac{1}{2 \ell+1}\right.$ tra $) I_{\ell}$, with $I_{\ell}$ the $(2 \ell+1) \times(2 \ell+1)$ identity matrix, and where $a^{0}=a-a \in s u(2 \ell+1)$.

Then the condition: $\tau_{\infty} \in \mathscr{L} \mathcal{O} \mathscr{B}$ can be reformulated by:

$\tau_{\infty} \in \mathscr{L} \mathscr{O B} \quad$ iff $\quad(\forall A \in \mathscr{C}): \quad \lim _{L \rightarrow \infty}(1 / L) \sum_{\ell \leqq L} \frac{1}{2 \ell+1}\left|J_{\ell}\left(\tau_{\ell}^{-1}\left(\pi_{\ell} A \pi_{\ell}\right) \tau_{\ell}\right)-\bar{\sigma}_{A} I_{\ell}\right|^{2}=0$.

In view of the Szegö limit formula,

$$
\frac{1}{2 \ell+1} \operatorname{tr} \pi_{\ell} A \pi_{\ell}=\bar{\sigma}_{A}+O_{A}\left(\ell^{-1}\right)
$$

So, we can further reformulate this condition as:

$$
\tau_{\infty} \in \mathscr{L} \mathscr{O B} \Leftrightarrow(\forall A \in \mathscr{C}): \quad \lim _{L \rightarrow \infty}(1 / L) \sum_{\ell \leqq L} \frac{1}{2 \ell+1}\left|J_{\ell}\left(\tau_{\ell}^{-1}\left(\pi_{\ell} A \pi_{\ell}\right)^{0} \tau_{\ell}\right)\right|^{2}=0 .
$$

To demonstrate the usefulness of this reformulation, let us first prove that, for a fixed $A \in \mathscr{C}$, there exists $\tau_{\infty} \in \mathscr{O} \mathscr{B}$ satisfying;

$$
\mid\left(\tau_{\ell}^{-1}\left(\left(\pi_{\ell} A \pi_{\ell}\right) \tau_{\ell} Y_{m}^{\ell}, Y_{m}^{\ell}\right)-\bar{\sigma}_{A} \mid=O_{A}\left(\ell^{-1}\right) \text { (uniformly in } m=-\ell, \ldots, \ell\right) .
$$

In fact, (2.9) follows from (2.7) and:

(2.10) Proposition. Let $\mathscr{P}_{\mu}$ be the image $J_{\ell}\left(\mathcal{O}_{\mu}\right)$ of the orbit $\mathcal{O}_{\mu}$ under the moment map (a convex polytope, see $[G-S]$ ). Let $d V_{\mu}$ be the measure on $\mathscr{P}_{\mu}$ which is the push forward under $J_{\ell}$ of the symplectic volume measure ${ }^{v_{\mu}}$ on $\mathcal{O}_{\mu}$. Then the center of mass of $\mathscr{P}_{\mu}$, relative to $d V_{\mu}$, is $\bar{\mu}$, where $\bar{\mu}=\left(\frac{1}{2 \ell+1} \sum_{i=1}^{2 \ell+1} \mu_{i}\right) I_{\ell}$.

Proof. Let $\mu^{0}=\mu-\bar{\mu}$ be the traceless part of $\mu ; \mu^{0} \in h_{\ell}^{0}$ (the Cartan subalgebra of su $(2 \ell+1)$. Obviously $\mathscr{P}_{\mu}=\mathscr{P}_{\mu^{0}}+\bar{\mu}$ (translation in $\left.h_{\ell}=h_{\ell}^{0} \oplus \mathbb{R}\right)$. The proposition is equivalent to: 0 is the center of mass of $\mathscr{P}_{\mu^{0}}$.

Thus, we claim:

$$
\int_{\mathscr{P}_{\mu^{0}}} H d V_{\mu^{0}}(H)=0 .
$$

Since $\mathscr{P}_{\mu^{0}}=J_{\ell}\left(\mathcal{O}_{\mu^{0}}\right)$ and $d V_{\mu^{0}}=J_{\ell_{*}} d v_{\mu^{0}},(2.11)$ is equivalent to:

$$
\int_{\mathcal{O}^{0}} J_{\ell}(\xi) d v_{\mu^{0}}(\xi)=0
$$

hence to

$$
\int_{S U(2 \ell+1)} J_{\ell}\left(\tau^{-1} \mu^{0} \tau\right) d \tau=0 \quad(d \tau=\text { Haar measure }) .
$$

But, clearly, $\int_{S U(2 \ell+1)} \tau^{-1} \mu^{0} \tau d \tau=0$. 
We now prove (2.9). In view of (2.7), it follows from

$$
(\forall A \in \mathscr{C})\left(\exists \tau_{\infty} \in \mathcal{O} \mathscr{B}\right)(\forall \ell): J_{\ell}\left(\tau_{\ell}^{-1}\left(\pi_{\ell} A \pi_{\ell}\right)^{0} \tau_{\ell}\right)=0,
$$

but this immediately follows from (2.10).

We now return to (2.8). Clearly this will involve the asymptotic dispersion from the mean of $\mathscr{P}_{\mu^{\ell}(A)}$ as $\ell \rightarrow \infty$. Among many possible formulations, the following seems quite convenient.

Fix $A \in \mathscr{C}$, and let $\left\{X_{\ell}^{A}\right\}$ denote the following sequence of positive random variables on $\mathscr{O} \mathscr{B}$ :

$$
X_{\ell}^{A}\left(\tau_{\infty}\right)=\left|J_{\ell}\left(\tau_{\ell}^{-1}\left(\pi_{\ell} A \pi_{\ell}\right) \tau_{\ell}\right)-\bar{\sigma}_{A} I_{\ell}\right|^{2}
$$

It is obvious that the $X_{\ell}^{A}$ are independent random variables (they involve different components of $\tau_{\infty}$ ). Further, the condition that $\tau_{\infty} \in \mathscr{L} \mathscr{O} \mathscr{B}$ is just that

$$
(\forall A \in \mathscr{C}) \lim _{L \rightarrow \infty}(1 / L) \sum_{\ell \leqq L} \frac{1}{2 \ell+1} X_{\ell}^{A}\left(\tau_{\infty}\right)=0 .
$$

The statement of Theorem (1.14) is that this holds for almost all $\tau_{\infty}$. This can be reduced to Kolmogorov's strong law of large numbers ([I], p. 188) once the expected values and variances of the $X_{\ell}^{A}$ are calculated asymptotically.

Let us denote by:

i) $E(X)=\int_{\mathcal{O} \mathscr{B}} X\left(\tau_{\infty}\right) d \mu_{\infty}\left(\tau_{\infty}\right)$

ii) $V(X)=E\left((X-E X)^{2}\right)$

the expected value, respectively the variance, of a random variable $X: \mathcal{O B} \rightarrow \mathbb{R}$.

Our main lemma is:

(2.15) Lemma. Fix $A \in \mathscr{C}$. Then:

$$
E\left(X_{\ell}^{A}\right)=\frac{1}{\operatorname{vol}\left(S^{*} S^{2}\right)} \int_{S^{*} S^{2}}\left|\sigma_{A}^{\text {ave }}(\zeta)-\bar{\sigma}_{A}\right|^{2} d \mu(\zeta)+O_{A}\left(\ell^{-1}\right)
$$

Proof. Evidently,

$$
E\left(X_{\ell}^{A}\right)=\frac{1}{\operatorname{vol} U(2 \ell+1)} \int_{U(2 \ell+1)}\left|J_{\ell}\left(\tau^{-1}\left(\pi_{\ell} A \pi_{\ell}\right) \tau\right)-\bar{\sigma}_{A} I_{\ell}\right|^{2} d \tau \quad(d \tau=\text { Haar measure })
$$

We first note that, using (2.7), we may replace $X_{\ell}^{A}$ by $Y_{\ell}^{A}$ where:

$$
Y_{\ell}^{A}\left(\tau_{\infty}\right) \stackrel{\text { def }}{=}\left|J_{\ell}\left(\tau_{\ell}^{-1}\left(\pi_{\ell} A \pi_{\ell}\right) \tau_{\ell}\right)-\overline{\pi_{\ell} A \pi_{\ell}}\right|^{2} .
$$

Indeed, $X_{\ell}^{A}\left(\tau_{\infty}\right)-Y_{\ell}^{A}\left(\tau_{\infty}\right)=(2 \ell+1)\left|\bar{\sigma}_{A}-\frac{1}{2 \ell+1} \operatorname{tr} \pi_{\ell} A \pi_{\ell}\right|^{2}=O_{A}(1 / \ell)$.

Second, we observe that

$$
\begin{aligned}
E\left(Y_{\ell}^{A}\right) & =\frac{1}{\operatorname{vol} U(2 \ell+1)} \int_{U(2 \ell+1)} \mid J_{\ell}\left(\tau^{-1} \mu^{\ell}(A) \tau-\left.\bar{\mu}^{\ell}\right|^{2} d \tau\right. \\
& =\frac{1}{\operatorname{vol} \mathcal{O}_{\mu^{\ell}(A)}} \int_{\mu^{\ell}(A)}\left|J_{\ell}(\xi)-\bar{\mu}\right|^{2} d v_{\mu}(\xi) .
\end{aligned}
$$


The identities in (2.18) suggest a way of calculating $E\left(Y_{\ell}^{A}\right)$ asymptotically.

Indeed, for any $\mu \in h_{\ell}$, let us set:

$$
\begin{aligned}
D_{2}^{2}(\mu-\bar{\mu}) & =\frac{1}{\operatorname{vol} U(2 \ell+1)} \int_{U(2 \ell+1)} \mid J_{\ell}\left(\tau^{-1} \mu \tau-\left.\bar{\mu}\right|^{2} d \tau\right. \\
& =\frac{1}{\operatorname{vol} \mathcal{O}_{\mu}} \int_{\mathcal{O}_{\mu}}\left|J_{\ell}(\xi)-\bar{\mu}\right|^{2} d v_{\mu}(\xi) .
\end{aligned}
$$

( $D_{2}$ is the $2^{\text {nd }}$ deviation of $\mathscr{P}_{\mu}$ from its mean).

Clearly, $D_{2}^{2}(\mu-\bar{\mu})$ is a symmetric, homogeneous polynomial of degree 2 in $\mu$. So there exist constants $a_{\ell}, b_{\ell}$ such that:

$$
D_{2}^{2}(\mu-\bar{\mu})=\frac{1}{2 \ell+1}\left(a_{\ell} S_{1}^{2}(\mu)+b_{\ell} S_{2}(\mu)\right)
$$

where $S_{p}(\mu)=\sum_{i=1}^{2 \ell+1} \mu_{i}^{p}$ is the $p^{\text {th }}$ power function. Also, clearly, $E\left(Y_{\ell}^{A}\right)=D_{2}^{2}\left(\mu^{\ell}(A)-\right.$ $\left.\bar{\mu}^{\ell}(A)\right)$. So we are reduced to calculating $\left\{a_{\ell}, b_{\ell}\right\}$ asymptotically. We claim:

$$
\begin{aligned}
& a_{\ell}=-\frac{1}{2 \ell+1}[1+O(1 / \ell)] \\
& b_{\ell}=1+O(1 / \ell)
\end{aligned},
$$

where the $O$-symbols are independent of any parameters.

Proof of (2.21). First, we plug $\mu=(1,1, \ldots, 1)$ into (2.20) and conclude:

$$
(2 \ell+1) a_{\ell}+b_{\ell}=0 .
$$

Next, we introduce the Fourier transform of the orbit $\mathcal{O}_{\mu}(\mu \in \operatorname{su}(2 \ell+1))$ :

$$
\mathscr{F}_{\mu}(H)=\frac{1}{\operatorname{vol}\left(\mathcal{O}_{\mu}\right)} \int_{\mathcal{O}_{\mu}} e^{i<j(\xi), H>} d v_{\mu}(\xi) \quad\left(H \in h_{\ell}^{0}\right) .
$$

Associated to $\langle, \quad\rangle$ is a gradient $\nabla$ and Laplacian $\Delta$. They satisfy:

$$
\begin{aligned}
-\Delta e^{i\langle\mu, H\rangle} & =|\mu|^{2} e^{i\langle\mu, H\rangle}, \\
(1 / i) \nabla e^{i\langle\mu, H\rangle} & =\mu e^{i\langle\mu, H\rangle} .
\end{aligned}
$$

Clearly:

$$
D_{2}^{2}(\mu-\bar{\mu})=-\left.\Delta \mathscr{F}_{\mu-\bar{\mu}}(H)\right|_{H=0} .
$$

To make use of (2.23), we invoke a well-known formula [G-S, Sect. 33]:

$$
\int_{\mathcal{G}_{\mu}} e^{i\langle j,(\xi), H\rangle} \frac{d v_{\mu}(\xi)}{(2 \pi)^{n}}=\sum_{w \in W} \frac{\varepsilon(w) e^{i\langle w \mu, H\rangle}}{\prod_{\alpha \in R_{+}}\langle\alpha, H\rangle},
$$

where $\mu \in h_{l}^{0}, n=\operatorname{dim} \mathcal{O}_{\mu}, R_{+}$is the set of positive roots for $\operatorname{su}(2 \ell+1)$, and $W$ is the Weyl group of the pair $\left(G, G_{\mu}\right)\left(G=S U(2 \ell+1), G_{\mu}=\right.$ stabilizer of $\left.\mu\right)$.

We will use (2.26) with $\mu=\delta$, where $\delta=(1 / 2) \sum_{\alpha \in R_{+}} \alpha$.

First, let us denote by $n(\mu, H)$, respectively $d(H)$, the numerator, respectively the denominator in (2.26): 
Quantum Ergodicity on the Sphere

i)

$$
\begin{aligned}
n(\mu, H) & =\sum_{w \in W} \varepsilon(w) e^{i\langle w \cdot \mu, H\rangle}, \\
d(H) & =\pi_{\alpha \in R_{+}}\langle\alpha, H\rangle .
\end{aligned}
$$

Let us also denote by $D(H)$ the Weyl denominator:

iii)

$$
D(H)=\prod_{\alpha \in R_{+}}\left(e^{\langle\alpha / 2, H\rangle}-e^{-\langle\alpha / 2, H\rangle}\right) .
$$

Evidently $d(H)$ is the term of order $\left|R_{+}\right|$in the Taylor expansion of $D(H)$. Further, by Weyl's denominator formula

$$
D(H)=\sum_{w \in W} \varepsilon(w) e^{\langle w \delta, H\rangle} .
$$

Thus:

$$
n(\delta, H)=D(H)
$$

Since $\bar{\delta}=0$, the thing to calculate is $-\left.\Delta\left(\frac{D(H)}{d(H)}\right)\right|_{H=0}$.

But

$$
\begin{aligned}
-\left.\Delta\left(\frac{D(H)}{d(H)}\right)\right|_{H=0} & =\left.(-\Delta) \prod_{\alpha \in R_{+}} \frac{2 \sin \langle\alpha / 2, H\rangle}{\langle\alpha, H\rangle}\right|_{H=0} \\
& =\left.(-\Delta) \prod_{\alpha \in R_{+}}\left(1-\frac{1}{3 !}\langle\alpha / 2, H\rangle^{2}+O\left(|H|^{3}\right)\right)\right|_{H=0} \\
& =\frac{1}{4 \cdot 3 !}\left(\left.\Delta \sum_{\alpha \in R_{+}}\langle\alpha, H\rangle^{2}\right|_{H=0}\right) \\
& \left.=\frac{2}{3 !}\left|R_{+}\right| \quad \text { as }|\alpha|^{2}=4 \text { for } \alpha \in R_{+}\right) .
\end{aligned}
$$

For $S U(2 \ell+1), R_{+}=\left\{e_{i}-e_{j}: i>j: e_{i}\left(\mu_{1}, \ldots, \mu_{2 \ell+1}\right)=\mu_{i}\right\}$. So $\left|R_{+}\right|=\ell(2 \ell+1)$ and $D_{2}^{2}(\delta)=(1 / 6) \ell(2 \ell+1)=\frac{1}{2 \ell+1}\left(a_{\ell} S_{1}^{2}(\delta)+b_{\ell} S_{2}(\delta)\right)$. But $\delta=\ell e_{1}+(\ell-1) e_{2}+\cdots+$ $e_{\ell}-e_{\ell+2}-\cdots-\ell e_{2 \ell+1}$, so $S_{2}(\delta)=2\left[1^{2}+2^{2}+\cdots+\ell^{2}\right]=\frac{\ell(\ell+1)(2 \ell+1)}{3}$; and, $S_{1}(\delta)=0$. Hence $D_{2}^{2}(\delta)=\frac{\ell(\ell+1)}{3} b_{\ell}=\frac{\ell(2 \ell+1)}{6}$. Thus, $b_{\ell}=1+0(1 / \ell)$. Combining with (2.22) we get (2.21).

This concludes our discussion of (2.21). Returning to (2.15)-(2.18), we see:

$$
\begin{aligned}
E\left(Y_{\ell}^{A}\right) & =\frac{1}{2 \ell+1}(1+O(1 / \ell)) S_{2}\left(\mu^{\ell}(A)\right)-\left(\frac{1}{2 \ell+1}\right)^{2}(1+O(1 / \ell)) S_{1}\left(\mu^{\ell}(A)\right)^{2} \\
& =\frac{1}{2 \ell+1} S_{2}\left(\mu^{\ell}(A)\right)-\left(\frac{1}{2 \ell+1}\right)^{2} S_{1}\left(\mu^{\ell}(A)\right)^{2}+O\left(\left\|\mu^{\ell}(A)\right\|_{\infty} 1 / \ell\right)^{2} .
\end{aligned}
$$

Obviously, $\left\|\mu^{\ell}(A)\right\|_{\infty}<\|A\|$ (operator norm); while by the Szegö Theorem, 
$\frac{1}{2 \ell+1} S_{2}\left(\mu^{\ell}(A)\right)=\frac{1}{\operatorname{vol}\left(S^{*}\left(S^{2}\right)\right)} \int_{S^{*}\left(S^{2}\right)}\left|\sigma_{A}^{\text {ave }}\right|^{2}+O_{A}(1 / \ell)$, and $\frac{1}{2 \ell+1} S_{1}\left(\mu^{\ell}(A)\right)=\bar{\sigma}_{A}+$ $O_{A}(1 / \ell)$. Lemma (2.15) follows.

We next recall Kolmogorov's strong law of large numbers. Let $\left\{f_{n}\right\} \subset L^{2}$ be an independent sequence, and let $S_{n}=\sum_{k=1}^{n} f_{k}$. Then:

(KSL) $\left\{V\left(f_{n}\right)\right\}$ bounded implies $(1 / n)\left[S_{n}-E\left(S_{n}\right)\right] \rightarrow 0$ (a.s.)

(almost surely). (See [I, Theorem 4.5.3]).

We set:

$$
f_{\ell} \stackrel{\text { def }}{=} \frac{1}{2 \ell+1} X_{\ell}^{A}
$$

So:

i)

$$
E\left(f_{\ell}\right)=\frac{1}{2 \ell+1} D_{2}^{2}\left(\sigma_{A}^{\mathrm{ave}}-\bar{\sigma}_{A}\right)+O_{A}\left(1 / \ell^{2}\right)
$$

(in an obvious notation);

ii)

$$
f_{\ell}\left(\tau_{\infty}\right) \leqq \frac{1}{2 \ell+1}\left|J_{\ell}\left(\tau_{\ell}^{-1}\left(\pi_{\ell} A \pi_{\ell}\right) \tau_{\ell}\right)-\bar{\sigma}_{A} I_{\ell}\right|^{2}=O_{A}(1) .
$$

Clearly $V\left(f_{\ell}\right)$ is bounded.

(KSL) therefore implies:

$$
\frac{1}{L} \sum_{\ell \leqq L}\left[\frac{1}{2 \ell+1} X_{\ell}^{A}\left(\tau_{\infty}\right)-\frac{1}{2 \ell+1} D_{2}^{2}\left(\sigma_{A}^{\mathrm{ave}}-\bar{\sigma}_{A}\right)\right] \rightarrow 0 \quad \text { (a.s.). }
$$

Hence,

$$
\frac{1}{L} \sum_{\ell \leqq L} \frac{1}{2 \ell+1} X_{\ell}^{A}\left(\tau_{\infty}\right) \rightarrow 0 \quad \text { (a.s.) }
$$

or equivalently,

$$
\frac{1}{N(L)} \sum_{\ell \leqq L} X_{\ell}^{A}\left(\tau_{\infty}\right) \rightarrow 0 \quad \text { (a.s.) }
$$

$\left(N(L)=\right.$ \# eigenvalues $\left.\leqq L^{2}\right)$. (2.36)-(2.37) is the criteria that $\tau_{\infty}$ be Liouville distributed, at least as regards $A \in \mathscr{C}$. To get rid of the dependence on $A$, we first set:

$$
\mathscr{L} \mathscr{O O B}_{A}=\left\{\tau_{\infty}: \frac{1}{N(L)} \sum_{\ell \leqq L} X_{\ell}^{A}\left(\tau_{\infty}\right) \rightarrow 0\right\} .
$$

We have just seen that $\mu_{\infty}\left(\mathscr{L} \mathcal{O B}{ }_{A}\right)=1$ for any fixed $A$.

But $\mathscr{L} \mathscr{O} \mathscr{B}_{A}$ only depends on the principal symbol $\sigma_{A}$. Further, choose an orthonormal basis $\left\{\varphi_{n}\right\}$ of $L^{2}\left(S^{*}\left(S^{2}\right), d \mu\right)$, so that linear combinations $\sum a_{n} \varphi_{n}$, with rapidly decaying $a_{n}$, are dense in $C\left(S^{*}\left(S^{2}\right)\right)$. It is straightforward to show that:

$$
\mathscr{L} \mathcal{O B}=\bigcap_{n} \mathscr{L} \mathscr{O B}_{\mathrm{Op}\left(\varphi_{n}\right)} \text {, }
$$


where $\operatorname{Op}\left(\varphi_{n}\right)$ is any $\Psi D O$ with principal symbol $\varphi_{n}$. Since each member at right has measure 1, we conclude our main theorem:

$$
\mu_{\infty}(\mathscr{L} \mathscr{O B})=1 \text {. }
$$

Acknowledgements. This paper was written while the author was visiting at the Institut Fourier in Grenoble. Thanks are due to Y. Colin de Verdiere for his support, and to C. Anne, M. Brion, G. Courtois and T. Kappeler for many helpful comments.

\section{References}

[B] Berry, M.V.: Regular and irregular semi-classical wavefunctions. J. Phys. A: Math. Gen. 10, 2083-2091 (1977)

[CdeV.1] Colin de Verdière, Y.: Quasi-modes sur les variétés riemannienes compactes. Invent. Math. 43, 15-52

[CdeV.2] Colin de Verdière, Y.: Ergodicité et fonctions propres du Laplacien. Sem. BonySjostrand-Meyer, 1984-1985, exposé no. XIII (1985)

[Gu] Guillemin, V.: Band asymptotics in two dimensions. Adv. Math. 42, 48-282 (1981)

[G-S] Guillemin, V., Sternberg, S.: Symplectic techniques in physics. Cambridge: Camb. U. Press, 1984

[HMR] Helffer, B., Martinez, A., Robert, D.: Ergodicité et limite semi-classique. Commun. Math. Phys. 109, 313-326 (1986)

[I] Ito, K.: Introduction to Probability Theory. Cambridge: Cambridge Univ. Press 1984

[ST] Schrader, R., Taylor, M.: Semi-classical asymptotics, gauge fields, and quantum chaos. J. Funct. Anal. 83, 258-316 (1989)

[Sn] Snirelman, A.I.: Ergodic properties of eigenfunctions. Usp. Math. Nank 29, 181-182 (1974)

[T] Terras, A.: Harmonic analysis on symmetric spaces and applications 1. Berlin, Heidelberg, New York: Springer 1985

[U] Uribe, A.: A Symbol calculus for a class of Pseudodifferential Operator on $S^{n}$ and band asymptotics. J. Funct. Anal. 59, 535-556 (1984)

[V] Voros, A.: Semi-classical ergodicity of quantum eigenstates in the Wigner representation. In: Stochastic Behaviour in Classical and Quantum Hamiltonian Systems. SLNP 93, 326-333 (1979)

[Wa] Walters, P.: An introduction to ergodic theory. Berlin, Heidelberg New York: Springer 1982

[Wei] Weinstein, A.: Asymptotics of eigenvalue clusters for the Laplacian plus a potential. Duke Math. J. 44, 883-892 (1977)

[Wi] Widom, H.: Eigenvalue distribution theorems for certain homogeneous spaces. J. Funct. Anal. 32 (1979)

[Z.1] Zelditch, S.: Quantum transition amplitudes for ergodic and for completely integrable systems. J. Fun. Anal. 94, 415-436 (1990)

[Z.2] Zelditch, S.: Uniform distribution of eigenfunctions on compact hyperbolic surfaces. Duke Math. J. 55, 919-941 (1987) 
